\title{
LES RYTHMES DE L'EXISTENCE
}

Véronique Verdier ${ }^{1}$

\section{RÉSUMÉ}

Cet article esquisse l'itinéraire de reconquête des rythmes de l'existence. Partant d'un constat, l'existence est en fait rythmée mais ces rythmes sont le plus souvent passivement subis et ne sont pas ceux choisis par le sujet, nous nous demandons comment retrouver une maîtrise de ces rythmes car cette maîtrise est une condition pour vivre mieux, sinon pour bien vivre. De l'écoute du corps, mis en péril par la rupture d'une maladie, en passant par la contrainte de règles rigides, nous verrons que l'issue se situe dans la création de rythmes propres.

MotS-ClÉs : existence, rythme, création, désir. Spinoza, Bachelard, Canguilhem, Lefebvre.

\footnotetext{
${ }^{1}$ Docteur en Philosophie, Véronique Verdier a une double formation philosophique et musicale. Elle est professeur en lycée et chercheuse associée au Centre d'Histoire des Philosophies Modernes (Université Paris 1). verdier.vero@free.fr http://veroniqueverdier.fr
} 
"Un botaniste qui bornerait sa science à dire que toutes les fleurs se fanent serait le digne émule du philosophe qui fonde sa doctrine en répétant: tout s'écoule et le temps fuit". Bachelard, La Dialectique de la durée

L'existence apparaît comme un flux, un mouvement permanent sur lequel il semble difficile d'avoir prise en raison de sa nature temporelle. Rien n'est stable, tout change toujours, tout ne cesse de passer. Seule une sortie hors du temps permettrait d'accéder à une certaine permanence. Rien de plus classique que cette opposition entre tenants d'un mobilisme universel et ceux de la permanence de l'Être, Héraclite contre Parménide, l'opposition est ancienne, antique même.

Suractivité, frénésie, accélération du temps propres à notre époque accentuent et renforcent ce sentiment d'un flux qui emporte tout. En réaction, émerge une timide volonté de décélération dont on ne sait encore si elle relève d'un effet de mode éphémère, voué à passer lui aussi. Mais s'agit-il seulement de ralentir et de faire la même chose, moins vite ? J'aimerais esquisser l'idée selon laquelle il est possible, au cœur même de l'existence, d'avoir prise sur ce flux, précisément par l'instauration de rythmes.

\section{Les RYthmes De LA Vie QuOtIDIENNE}

Envisager l'existence comme un mouvement permanent relève d'une conception continuiste du temps, conception qui s'accommode mal de l'idée de rythme puisqu'un rythme se constitue d'une alternance de mouvement et de repos. Or, "s'arrêter de couler serait s'arrêter de subsister. S'immobiliser, c'est mourir" (BACHELARD, 1993, p. 2)². Arrêt, interruption, repos seraient toujours provoqués de l'extérieur et synonymes d'une forme de déchéance, le vivant sombrant à ces occasions dans l'inertie matérielle.

Cependant, penser la temporalité comme un flux continu ne permet pas de rendre compte de la diversité temporelle des phénomènes vécus. Bachelard souligne : "la nécessité de fonder la vie complexe sur une pluralité de durées qui n'ont ni le même rythme, ni la même solidité d'enchaînement, ni la même puissance de continu" (BACHELARD, 1993, p. VIII). Ces diverses durées expriment une variété de rythmes.

2 Bergson est ici explicitement visé : "Disons tout de suite que du bergsonisme nous acceptons presque tout, sauf la continuité !" (Bachelard, 1950, p.- 7). 
Loin d'être un long flux indistinct, la vie dans sa plus simple quotidienneté est rythmée. Le corps, déjà, dicte ses rythmes, et ce, dès la naissance: respiration, pulsation cardiaque, veille et sommeil, alimentation et digestion. Ces rythmes physiologiques sont basiques, au sens où ils forment la base même de la vie et la possibilité de sa continuation, ils constituent d'ailleurs l'essentiel de la vie quotidienne du nouveau-né, une vie qui se déroule comme une épure du rythme.

La nature dicte aussi ses rythmes, ceux des saisons qui sont cycliques et suivent ce que Bachelard appelle joliment le calendrier des fruits : "Que nous ayons un intérêt physique à nous adapter très rigoureusement aux rythmes végétaux, c'est ce qui est de plus en plus évident depuis qu'on connaît la spécificité des vitamines: I'heure de la fraise, l'heure de la pêche et du raisin sont des occasions de renouveau physique, d'abord avec le printemps et l'automne" (BACHELARD, 1993, p. 147). Ne pas les respecter, c'est prendre le risque de vivre à contretemps.

Certes, mais on le sait, très vite, très tôt, I'humain est pris dans les mailles du culturel. Les heures de repas résultent elles-mêmes de conventions sociales, qui produisent des habitudes constituant comme une seconde nature : on finit par avoir faim à ces heures-là. Et c'est la société qui dicte alors ses propres rythmes. Le temps social, mesuré au moyen de montres et d'horloges, règle les différents aspects de la vie quotidienne : "les heures du sommeil et du réveil, les heures des repas et de la vie privée, les relations des adultes avec les enfants, les distractions et les loisirs, les rapports au lieu d'habitation" (LEFEBVRE et REGULIER, 1985, p. 191).

Dans les sociétés industrielles centrées sur la référence ultime qu'est le travail, la répétition d'activités qui s'inscrivent dans un temps linéaire peut être " lassante, épuisante, fastidieuse, alors que le retour d'un cycle a l'allure d'un avènement et d'un événement. Son début qui n'est pourtant qu'un recommencement, a toujours la fraîcheur d'une découverte et d'une invention. L'aube a toujours un charme miraculeux, la faim, la soif se renouvellent merveilleusement...." (LEFEBVRE et REGULIER, 1985,p. 191). Le cyclique, bien qu'absorbé voire ignoré par les sociétés modernes, est un résidu du cosmique et du naturel en l'homme encore prégnant dans la vie quotidienne ${ }^{3}$.

3 L'ancrage de la vie des femmes dans des cycles menstruels, par exemple, participe d'une certaine manière d'appréhender la temporalité. 
II règne en somme une lutte entre rythmes personnels et processus liés à l'organisation socio-économique de la production. "Par exemple, les activités nocturnes se multiplient, bouleversant les rythmes circadiens. Comme si la journée ne suffisait pas à accomplir les tâches répétitives, la pratique sociale mord peu à peu sur la nuit" (LEFEBVRE et REGULIER, 1985, p. 192). Les rythmes biologiques s'en trouvent modifiés et profondément altérés.

\section{Des RYthmes Subis}

Qu'est-ce au juste qu'un rythme ? Une alternance entre mouvement et repos, avons-nous dit. Henri Lefebvre et Catherine Régulier précisent : "pour qu'il y ait rythme, il faut qu'il y ait répétition dans un mouvement, mais pas n'importe quelle répétition" (LEFEBVRE et REGULIER, 1985, p. 194). Le retour monotone et implacable d'un même bruit, la goutte d'eau du robinet qui fuit, le tic-tac du réveil, ne forme pas un rythme. Pour qu'il y ait rythme, il faut qu'il y ait des contrastes, des arrêts, des temps forts et des temps faibles, dit-on en musique, ainsi que des reprises. "Donc une mesure, mais une mesure interne, qui se distingue fortement sans pourtant s'en séparer de la mesure externe, le temps $t$ (temps de l'horloge ou du métronome)" (LEFEBVRE et REGULIER, 1985, p. 194). Un rythme exprime un mouvement non mécanique. "Le rythme comporte un temps différencié, une durée qualifiée" (LEFEBVRE et REGULIER, 1985, p. 194). En ce sens, le rythme est la marque même du vivant, il désigne le devenir tel qu'il est vécu ${ }^{4}$.

Mais le plus souvent, les rythmes de l'existence sont imposés de l'extérieur et tributaires de la vie en société : la famille, l'école, le monde du travail. Et ces rythmes, auxquels il faut se plier, se soucient fort peu d'être en harmonie ou en accord avec ce que chacun pourrait intimement désirer. À tel point qu'ils s'annulent alors comme rythmes.

Avec la répétition inéluctable d'un emploi du temps non choisi, la vie s'écoule semblable à elle-même, sans contraste, telle une mécanique plus ou moins bien huilée qui broie l'individu. Dans Le Premier homme, Camus décrit le travail de bureau sans éclat de son personnage principal : "l'ennui, la fatigue lui révélaient sa malédiction, celle du travail bête à pleurer dont la monotonie interminable parvient à rendre en

4 Une différence semblable existe entre la programmation mécanique d'une boîte à rythmes et le jeu vivant d'un percussionniste. 
même temps les jours trop longs et la vie trop courte" (CAMUS, 1994, p. 248). Paradoxalement, le temps semble ne plus s'écouler car les journées n'en finissent pas de finir, et la vie passer trop vite car elle ne laisse prise à aucune activité signifiante. Le danger est grand d'être saisi d'une certaine torpeur en se sentant aussi peu vivant.

Certains savent toutefois se satisfaire d'une vie routinière toujours identique à elle-même, ils y gagnent l'assurance d'une vie sans aspérité et sans surprise. D'autres éprouvent en se surinvestissant dans leur travail une forme de toute-puissance, toutepuissance illusoire car l'adaptabilité forcenée et la mobilité permanente, requises par un certain type de management, exacerbent la vacuité d'une telle fuite en avant.

Face à cela, la vie privée ne constitue pas toujours un îlot favorisant l'expression de rythmes à soi. La vie matérielle est certes facilitée par les progrès techniques: machines à laver, aspirateur, robots allègent les tâches ménagères, mais contribuent aussi, par une succession d'actes sans liens les uns avec les autres, à un morcellement du temps 5 .

Bien sûr, la vie privée ne saurait se réduire à un empilement de tâches ménagères. Mais c'est à présent le numérique, sous la forme de divers écrans, qui accapare le reste de temps disponible. En particulier le smartphone, prolongement de l'individu contemporain, rend accessible, de façon quasi instantanée, informations, photographies, musiques, films, jeux, réseaux sociaux. Cette accessibilité n'est pas sans incidence, elle suscite en particulier une certaine impatience. Personne ne peut plus attendre, chacun souhaite de manière impulsive accéder à tout, sans aucun délai ${ }^{6}$. Cet usage frénétique du numérique produit une saturation, chacun se retrouvant sous stimulation permanente, sans respiration et sans temps mort.

Ces situations ont pour dénominateur commun de provoquer une dépossession du soi. Mais est-il possible d'échapper à un tel phénomène et de se réapproprier les rythmes de son existence?

On le sait, un élément nouveau peut faire vaciller n'importe quelle construction aussi solide soit-elle, prouvant ainsi qu'elle n'est en réalité ni nécessaire, ni immuable. Devenir amoureux, par exemple, fait basculer dans une autre quotidienneté, cette

5 Ces tâches incombent encore largement aux femmes qui les délèguent quand elles le peuvent et si elles le veulent à des... femmes de ménage.

6 Le Monde a constaté qu'après cinq secondes d'attente sur son site, trois lecteurs sur dix abandonnaient. En réduisant ce temps de connexion à deux secondes, il en a accru la fréquentation. 
irruption de nouveauté produisant une reconfiguration dynamique du quotidien le plus répétitif. Cela signifie qu'il est possible, lorsqu'on le désire, d'agir sur ces rythmes et de les transformer.

La rythmanalyse propose de prévenir les souffrances psychologiques par une vie eurythmique qui suivrait de "bons" rythmes grâce à une thérapeutique du corps ${ }^{7}$. Elle se distingue de la psychanalyse dans la mesure où elle ne s'intéresse qu'indirectement au psychisme. Pour Lefebvre, le rythmanalyste "écoute et d'abord son corps ; il y apprend les rythmes, pour ensuite apprécier les rythmes externes. Son corps lui sert de métronome" (LEFEBVRE, 1992, p. 32). II recourt à des techniques ancestrales : maîtrise de la respiration, du cœur, de l'utilisation synchronique des mouvements dans la danse. Cette attention à ses propres rythmes, aux impressions de ses cinq sens, doit permettre de se défaire des durées mal ajustées, de se rééquilibrer et d'atteindre ainsi une forme de bien-être ${ }^{8}$.

Bachelard envisage aussi un retour au corps afin de lutter contre les arythmies ou les rythmes mal faits. II s'agit par des exercices de remettre en valeur et de régulariser les "grands rythmes naturels qui soutiennent la vie. Et d'abord la respiration, lente et régulière cadence qui marque profondément, quand on l'a bien libérée de tout souci organique, notre confiance temporelle" (BACHELARD, 1993, p. 146). La régularité du souffle permet par exemple de dominer une douleur dentaire : "Il suffit par une attention calme de ramener à ses proportions précises, d'éviter l'agacement général, l'agitation générale, qui viendraient emplir les intervalles de la douleur précise" (BACHELARD, 1993, p. 146). Ce rythme respiratoire, repris de doctrines indiennes, permet une coordination de tout l'organisme. Un rythme vital rapide est troublé, on y remédie par l'encadrement d'un rythme plus lent. Ralentir ! Telle semble être l'invitation de cet enseignement pour instituer une régularité pacifiée.

Mais Bachelard remarque à juste titre que la pensée ou l'esprit sont dans cette affaire quelque peu laissés de côté. L'esprit a besoin en lui-même et pour lui-même d'autres repères. " Et si, comme nous le croyons, la vie intellectuelle doit devenir physiquement parlant - la vie dominante, si le temps pensé doit dominer le temps vécu, il faut s'attacher à la recherche d'un repos actif qui ne peut se satisfaire des dons

7 La rythmanalyse est élaborée par le philosophe portugais Lùcio Alberto Pinheiro dos Santos, exilé au Brésil. Ses idées seront reprises et développées en particulier par Gaston Bachelard et Henri Lefebvre. 8 Se réapproprier son corps a, dans la perspective marxiste de Lefebvre, une incidence sociologique et urbaine, cette assise individuelle constituant une étape nécessaire pour produire des transformations concrètes du monde. 
gratuits de l'heure et de la saison" (BACHELARD, 1993, p. 148). II faut aussi chercher une élaboration spirituelle, au sens où il faut "redonner à l'esprit la maîtrise des dialectiques de la durée" (BACHELARD, 1993, p. 150). Car il ne saurait suffire d'être à l'écoute de ses seuls rythmes corporels. Mener une vie humaine, c'est s'affirmer pardelà la dimension biologique du vivant, c'est vouloir plus qu'un corps en bonne santé, même si cette santé est une condition nécessaire au déploiement de son existence. La preuve, la maladie apparaît très souvent comme une véritable rupture de rythme.

\section{RUPTURES DE RYTHME}

Séisme, catastrophe nucléaire, acte terroriste, viol, la sidération d'un événement brutal et traumatisant laisse hagard, le déroulement même de la vie semble alors comme suspendu. Plus question de bons ou de mauvais rythmes, de rythmes subis ou choisis, c'est vivre qui semble la difficulté incontournable. S'accrocher à la pure vitalité de la vie, assurer ses fonctions vitales pour persévérer dans l'être est la seule alternative à se laisser dépérir, une étape nécessaire avant de pouvoir envisager de se reconstruire.

Dans un autre registre, lors de la pandémie de coronavirus de 2020, le confinement a provoqué une interruption radicale et durable des rythmes habituels. Pour éviter tout contact, écoles, universités, musées, salles de concert, bibliothèques, restaurants et autres activités jugées "non nécessaires" ont été mis à l'arrêt. Confronté à la perte de ses repères familiers, isolé et replié chez lui, chacun a dû bricoler à la hâte d'autres rythmes pour tenir et ne pas sombrer dans le désarroi, la détresse ou la dépression. Si le recours au numérique a permis de maintenir un lien social, il a aussi fait sentir combien le lien physique et charnel avec autrui est précisément indispensable. À cet égard, les inégalités sont apparues encore plus criantes, la plupart n'ayant ni les conditions matérielles, ni les ressources personnelles pour vivre une telle épreuve ${ }^{9}$.

\footnotetext{
9 Cette expérience a toutefois présenté des aspects inattendus : le calme et le silence des grandes villes, une diminution de la pollution, une décroissance et une relocalisation forcées, et surtout l'abandon immédiat, mais assurément temporaire, des politiques d'austérité budgétaire, causes de la gravité de la crise sanitaire. Si ces politiques n'ont pas provoqué la pandémie, l'impossibilité pour les systèmes de santé publique de prendre en charge l'afflux massif de patients est bien la conséquence directe de ces politiques. En France, le management entrepreneurial à la manœuvre dans les hôpitaux depuis une vingtaine d'années a produit une baisse de $30 \%$ du nombre de lits par habitant, plaçant ainsi la France loin derrière l'Allemagne, le Japon ou la Corée du Sud (Velut, 2020). La pénurie dramatique de masques, de tests et de désinfectants est bien le fait de décideurs imprévoyants et irresponsables. Si
} 
Si de telles situations sont exceptionnelles, l'épreuve plus courante de la maladie est largement partagée : fracture immobilisante, hospitalisation éprouvante, opération aux lourdes conséquences, toutes signent une rupture de rythme.

La maladie, qu'elle soit organique ou psychologique, a longtemps été conçue comme une rupture de normalité et la guérison comme un retour à la normale. Canguilhem envisage les choses autrement. Les fonctions d'un organisme vivant sont interdépendantes, leurs rythmes sont accordés, la maladie en est un dysfonctionnement, elle est une autre manière de vivre. La maladie est "vraiment pour le malade une autre allure de la vie. [...] L'état pathologique n'est pas un simple prolongement, quantitativement varié, de l'état physiologique, il est bien autre" (CANGUILHEM, 1998, p. 51). C'est un jeu nouveau de l'organisme, un remaniement radical. "La maladie est une expérience d'innovation positive du vivant et non plus seulement un fait diminutif ou multiplicatif" (CANGUILHEM, 1998, p. 122). Que toute nouveauté ne soit pas bonne à vivre, il faut tout de même faire avec lorsque surgit la maladie.

Si la maladie se définit comme altération anatomique, comme trouble physiologique ou psychologique, c'est toutefois le malade qui se saisit comme tel. " La médecine existe comme art de la vie parce que le vivant humain qualifie lui-même comme pathologiques, donc comme devant être évités ou corrigés, certains états ou comportements appréhendés sous forme de valeur négative" (CANGUILHEM, 1998, p. 77). Malgré le développement des techniques d'investigation, ce n'est pas d'abord au niveau des tissus et des cellules que s'appréhende la maladie, mais du point de vue de la conscience du sujet concerné ${ }^{10}$. "C'est donc bien toujours en droit, sinon actuellement en fait, parce qu'il y a des hommes qui se sentent malades qu'il y a une médecine" (CANGUILHEM, 1998, pp. 53-54). D'où l'aspect sournois de certains cancers ou de dissociations psychiques qui n'apparaissent que trop tardivement voire

depuis le 12 mars 2020, la santé n'a pas de prix pour le Président Emmanuel Macron, elle en avait un, bien trop lourd, jusqu'au 11 mars. Et ce n'est pas la Main invisible du marché et le ruissellement qui sont venus à la rescousse!

10 La confiance absolue dans les imageries médicales entraîne une mise entre parenthèses de l'individu malade. Didier Sicard rapporte le témoignage suivant : "Rony Braumann raconte que, paralysé des quatre membres après une intervention, il voyait avec angoisse les médecins le rassurer sur cette absence de paralysie parce que les indicateurs chiffrés témoignaient du fait qu'il allait bien" (Sicard, 2007, p. 134). Les analyses se substituent alors jusqu'à l'absurde au vécu et au perçu du sujet. 
jamais à la conscience du malade ${ }^{11}$. Mais le plus souvent, le malade a conscience d'être malade parce qu'il éprouve une douleur ou un mal-être. Et il sait que cette douleur n'est pas normale. C'est elle qui exprime la nouveauté physiologique ou psychologique que doit reconnaître et prendre au sérieux le sujet lui-même, son entourage ainsi que le thérapeute.

C'est un fait, la vie n'est pas identique à elle-même dans la santé et dans la maladie. "La frontière entre le normal et le pathologique est parfaitement précise pour un seul et même individu considéré successivement" (CANGUILHEM, 1998, pp. 119120). La maladie perturbe le déroulement de la vie qui ne marche plus aux mêmes rythmes, elle fait souffrir et suscite une forme d'angoisse lorsqu'elle est grave. Elle fait perdre au vivant ses capacités d'adaptation aux variations du milieu, aux écarts et aux changements, la capacité d'instituer d'autres normes dans d'autres conditions, capacités qui caractérisent une vie en bonne santé ${ }^{12}$.

Mais la guérison n'est pas pour autant un retour à la normale car la vie ne connaît littéralement pas de retour en arrière, de réversibilité, elle est toujours un mouvement en avant. "La vie admet des réparations qui sont vraiment des innovations physiologiques. La réduction plus ou moins grande de ces possibilités d'innovation mesure la gravité de la maladie" (CANGUILHEM, 1966, p. 129). Guérir c'est se donner de nouvelles normes de vie, parfois supérieures aux anciennes, mais jamais identiques à elles. Quelqu'un ayant risqué l'amputation d'un bras ou d'un autre membre sera heureux d'en recouvrer n'importe quel usage après son intervention.

Être guéri, c'est pouvoir reprendre une activité interrompue par la maladie qui ira désormais à un autre rythme: "Même si cette activité est réduite, même si les comportements possibles sont moins variés, moins souples qu'ils n'étaient auparavant, l'individu n'y regarde pas toujours de si près. L'essentiel est d'être remonté d'un abîme d'impotence ou de souffrance où le malade a failli rester ; l'essentiel est de

11 En psychiatrie, de nombreux troubles (délire de persécution, hallucination, anosognosie,...) ne sont pas saisis comme tels par le sujet.

12 Ces analyses sont confortées par les travaux des médecins Jeffrey Hall, Michael Rosbach et Michael Young, prix Nobel de médecine en 2017, qui mettent en évidence le rôle des rythmes chronobiologiques dans le fonctionnement du vivant. Les cycles circadiens d'un organisme sont réglés par des mécanismes moléculaires déterminés. Leur trouble peut causer des maladies telles que l'obésité, le diabète, l'hypertension ou encore des cancers. La prise en charge de ces maladies devrait elle-même tenir compte des rythmes physiologiques propres à chacun. La voie prometteuse de la chronothérapeutique consiste à ajuster l'administration d'un traitement à ces rythmes. En chimiothérapie, par exemple, l'efficacité des médicaments s'en trouve démultipliée et les effets indésirables limités. 
l'avoir échappé belle" (CANGUILHEM, 1998, p. 73). On peut vivre avec un seul rein, même s'il faut alors se ménager, car on ne peut plus se permettre de perdre cet unique rein. La guérison reconstruit en quelque sorte une nouvelle entité.

Ce qui permet de comprendre par contraste ce qu'est la santé. "Être en bonne santé, c'est pouvoir tomber malade et s'en relever, c'est un luxe biologique" (CANGUILHEM, 1998, p. 132). La vie au sens physiologique est surabondance de moyens, elle a toujours trop de capacités, trop de neurones, trop de reins, trop de poumons, trop de pancréas, cette prodigalité permet l'excès : "l'abus possible de la santé fait partie de la santé" (CANGUILHEM, 1998, p. 133). La vie est capacité à tolérer des infractions à la norme habituelle, à surmonter des crises. "La vie n'est donc pas pour le vivant une déduction monotone, un mouvement rectiligne, elle ignore la rigidité géométrique, elle est débat ou explication avec un milieu où il y a des fuites, des trous, des dérobades et des résistances inattendues" (CANGUILHEM, 1998, p. 131). Cette confrontation à ce qui fait événement construit peu à peu l'histoire d'un être vivant. Et dans cette histoire "la santé c'est un ensemble de sécurités et d'assurances, sécurités dans le présent et assurances pour l'avenir" (CANGUILHEM, 1998, p. 131). Une solide assise pour l'existence.

La santé, donnée ou reconquise, c'est, selon la définition de Leriche, la vie dans le silence des organes. Un état où l'on remarque le corps le moins possible en dehors d'un joyeux sentiment d'existence, comme le précise Jaspers (CANGUILHEM, 1998, p. 74). Mais ce sentiment ne relève pas seulement d'un corps en bonne santé, il y faut une reprise en mains de son existence par le sujet lui-même.

\section{Des Rythmes (A MARChe) Forces}

Certaines reprises en mains s'avèrent être des impasses, tel est le cas de l'engouement pour des régimes alimentaires drastiques, médicalement non motivés: le sans gluten, le sans lactose, le tout végétarien, le tout vegan. La norme fluctue, pardelà toute rationalité, au même rythme que les modes. L'individu pense ainsi maîtriser son existence en réglant la difficile et lancinante question qui se pose au quotidien de savoir ce qu'il désire manger. Question qu'il esquive en lui substituant celle de ce qu'il peut ou ne peut pas faire, ou plutôt de ce qu'il doit ou ne doit pas faire, s'imposant par là une forme d'hygiénisme.

L'hygiénisme, de façon générale, se réduit à une simple gestion du corps : "règles d'hygiène, exercices corporels, alimentation saine, précautions à prendre pour 
éviter les maladies sexuellement transmissibles, soumission aux prescriptions du médecin. Ne pas fumer, ne guère boire, ne pas prendre de risques, vivre sagement, c'est-à-dire précautionneusement" (THOMAS, 2013, p. 167). La retenue est certes bonne, mais elle ne peut tenir lieu de conduite de l'existence. Jean-Paul Thomas l'écrit: " Le bien-être organique n'est pas l'alpha et l'oméga d'une vie humaine" (THOMAS, 2013, p. 168). Que penserait-on d'une éducation qui recommanderait exclusivement de manger et dormir raisonnablement?

Cette attitude est même contre-productive car elle "développe, sous couvert de préceptes triviaux, une posture précautionneuse, une sorte de méfiance généralisée à l'égard du risque de vivre" (THOMAS, 2013, p. 167). Elle n'aide pas l'homme à mieux vivre ou à bien vivre, elle tend à réduire l'humanité à la préservation d'un " patrimoine corporel, menacé par les excès, les addictions, les souffrances et les échecs que seule une vie totalement dénuée de passions et d'élan créateur peut garantir" (THOMAS, 2013, p. 16). Une telle vie, frileuse et en réalité fragile, renforce le repli sur soi d'individus angoissés car obnubilés par la préservation de ce qu'une économie néolibérale ose appeler leur capital-santé.

La reprise en mains des rythmes de l'existence peut aussi s'opérer en s'appuyant fermement sur des préceptes religieux, non pas simplement des rituels, des fêtes cultuelles, des règles que chacun adapte de fait en se les appropriant, mais bien plutôt sur une orthopraxie qui domine les courants fondamentalistes ultrarigoristes. L'orthopraxie prétend déterminer la conduite individuelle de la vie quotidienne d'une manière strictement conforme à des prescriptions religieuses. Elle ne se contente pas d'élaborer des commandements généraux, elle établit et fixe dans leurs moindres détails ce qui est interdit, ce qui est proscrit, ce qu'il faut faire et comment le faire. Tout doit être précisé : chaque action, chaque comportement, chaque attitude, chaque rapport à l'autre en toute situation.

De façon quasi-obsessionnelle, ces prescriptions sont particulièrement pressantes en direction des femmes. Le salafisme, parmi d'autres, leur impose des diktats vestimentaires fondés sur l'interdit de montrer en public la quasi-intégralité de leur corps. Les haredims pénètrent, eux, dans la sphère privée. La femme, impure, ne doit pas frôler son propre époux pendant ses menstruations, même par inadvertance. Certaines mouvances vont jusqu'à fixer le nombre licite de baisers, leur moment et leur 
durée lors d'unions sexuelles dont les fréquences sont elle-mêmes calculées, codifiant ainsi une intimité qui pourrait échapper au champ de l'intégrisme religieux ${ }^{13}$.

La profusion et la rigidité de règles et d'interdits pourtant difficiles à intégrer et donc à respecter est perçue comme rassurante. Le rigorisme, quel que soit son fondement (religieux, mystique, New Age), instille une confiance aveugle, voire une certaine arrogance dans l'esprit de ses adeptes qui se perçoivent comme les plus purs, voire les meilleurs, car seuls capables de se préserver et de résister aux multiples agressions d'un monde conçu comme perverti ou décadent.

Simmel souligne les risques individuels de telles prescriptions: "maints impératifs éthiques comportent un idéal d'une perfection si rigide qu'il ne permet pas d'actualiser à partir de lui des énergies susceptibles d'être accueillies par nous dans notre développement" (SIMMEL, 1988, p. 196). Ces impératifs ne peuvent pas aider à orienter et conduire l'existence, ils n'ont paradoxalement aucune efficacité car ils demeurent extérieurs aux sujets, à leur périphérie. L'individu y est nié en tant qu'individu, et c'est peut-être en somme ce qu'il désire.

En se fondant sur des normes externes si contraignantes, le sujet refuse de s'engager dans son histoire personnelle, il se décharge des aléas d'une vie qui repose toujours plus ou moins, qu'il le veuille ou non, sur l'initiative personnelle. Loin de se reprendre en mains, le sujet se retrouve en réalité ici aussi dépossédé de lui-même, comme si tel était d'ailleurs l'objet de ces quêtes: se détourner, se délester, se démettre de soi. Mais un individu ne peut vivre à ces rythmes forcés car de tels rythmes ne sont tout simplement pas vivables.

\section{La Creation De Rythmes Propres}

Il faut partir ou repartir de soi et être à l'origine de ses propres rythmes. "Nous ne serons des êtres fortement constitués, vivant dans un repos bien assuré, que si nous savons vivre sur notre propre rythme, en retrouvant, à notre gré, à la moindre fatigue, au moindre désespoir, l'impulsion de nos origines" (BACHELARD, 1993, IX). Instituer ce rythme fondamental de l'existence nécessite de s'approprier les différents rythmes des différents actes dans lesquels on s'engage.

13 Autre cible privilégiée, lorsqu'il n'est pas condamné à la peine de mort, l'homosexuel : un homme ayant une démarche "efféminée" (les clichés ont la vie dure) apprendra comment mouvoir son corps et discipliner une certaine tenue des poignets (Geoffroy, 2013). 
Mais l'existence, et pas seulement la vie biologique, se caractérise par une surabondance vertigineuse : trop de possibilités, trop de perspectives, trop de sollicitations diverses et variées. Face à cela, il faut choisir, et nombreux sont ceux qui tentent d'éluder cette question du choix. Certes, chacun fait des choix, au moins a minima: une filière d'études menant à un métier qui s'accompagne de quelque divertissement. Mais ce sont trop souvent les circonstances ou les autres qui décident à la place du sujet. Ou plutôt le sujet décide que les événements, la famille, le destin, un idéologue décideront pour lui.

Repartir de soi, c'est décider de recommencer à soi. Mais qu'est-ce faire au juste ? C'est déjà s'émanciper des stéréotypes et cesser de subir autant qu'il est possible ce qui est imposé de l'extérieur, c'est aussi rompre avec les modalités habituelles, non réfléchies, de la vie quotidienne et gagner peu à peu en autonomie. C'est décider d'opérer des choix en étant à l'écoute de soi, c'est-à-dire non pas seulement à l'écoute de son corps comme phénomène biologique, mais à l'écoute de son désir. La question centrale autour de laquelle tourne la question des rythmes et que l'on peut passer toute sa vie à éviter est celle de ce que l'on désire être. La mesure interne à laquelle accorder les rythmes de son existence, c'est en effet le désir, un désir qui pourrait définir le sujet en propre et dans lequel il se reconnaîtrait.

Le sujet doit se choisir, se définir non en négatif, par tout ce qu'il aurait pu ou dû faire, qu'il n'a pas fait et qu'il regrette de ne pas avoir fait, mais en positif, par tout ce qu'il fait et désire faire. Comme l'écrit Robert Misrahi, "chaque action est une énergie et une temporalité" (MISRAHI, 1996, p. 451), elle ne laisse pas l'individu indemne. Celui-ci ne saurait dépenser sans compter et s'éparpiller en toutes directions. "Le sujet humain est un individu fini [...]. II ne peut être à la fois philosophe, poète, médecin, ingénieur, juriste, politique et enseignant" (MISRAHi, 1996, p. 451); pas plus que conducteur de bus, vétérinaire, astronaute, instituteur, pompier, chanteur ou toute autre combinaison comme en rêvent les enfants. Un sujet ne peut réaliser et déployer pleinement que certaines possibilités à l'exclusion des autres. Pour cesser de papillonner, il doit au moins une fois en sa vie se poser sérieusement cette question du choix qui n'est autre que celle de la construction de soi. On le comprend, il faut y réfléchir, s'examiner soi-même, éclairer son désir par la réflexion, c'est ainsi que l'esprit pourra maîtriser, comme l'écrit Bachelard, les dialectiques de la durée. L'essentiel est de trouver par où commencer ou recommencer, c'est la condition sine qua non afin 
que des actes, des engagements, des pensées, des désirs soient enfin, autant qu'il est possible, les siens.

Chaque activité a ses propres rythmes, et l'articulation des différents rythmes de l'existence est une tâche éminemment personnelle qui réclame un investissement de la part du sujet. Celui-ci doit s'organiser, hiérarchiser, faire passer l'essentiel avant l'accessoire ; l'inverse de ce que l'on fait habituellement. S'il a le privilège de travailler dans de bonnes conditions, il doit encore établir les modalités courantes de la vie quotidienne, s'entretenir, consacrer du temps à ses amis, laisser place à quelque passion, se divertir aussi, prendre des vacances pour vivre à un autre rythme : détente absolue, bricolage, lectures intenses, voyages. Ces différentes activités ne se juxtaposant plus de façon hasardeuse ou incohérente, une délicate alchimie peut s'établir entre elles, et le sujet jongler harmonieusement avec les changements de rythmes qu'elles impliquent.

Certains choix entraînent un mode de vie strictement régulé, c'est le cas par exemple du sportif de haut niveau ou de l'instrumentiste ; ceux-ci se plient à une hygiène de vie, à un entraînement quotidien exigeant, dont la durée et le moment optimaux doivent être fixés de manière adaptée ${ }^{14}$. D'un point de vue extérieur, une telle vie semble relever de l'abnégation tant elle est astreinte au rythme répétitif des mêmes schémas et des mêmes exercices. Cette apparente répétition dissimule en réalité un travail d'orfèvre dans la recherche de la maîtrise d'un geste, d'un son, d'une nuance. Retrouver le stade ou son instrument, c'est une fête quotidienne perpétuellement réactivée car il s'agit chaque jour, non pas seulement de retrouver les repères de la veille, mais de les affiner, de les améliorer et de les perfectionner, de faire mieux, en somme, et non pas de répéter indéfiniment la même chose ${ }^{15}$.

Lorsqu'ils sont choisis, et non subis ou forcés, les différents rythmes impulsés par les différentes activités constituent une existence qui ne s'éprouve pas comme un mouvement linéaire et mécanique. "Le rythme est vraiment la seule manière de discipliner et de préserver les énergies les plus diverses. II est la base de la dynamique vitale et de la dynamique psychique" (BACHELARD, 1993, p. 128). Cette dynamique, existentielle, prend la forme d'un renouvellement. Avec elle, on a envie de se lever le

14 il faut environ dix mille heures pour maitriser un instrument, ce qui represente trois heures de pratique quotidienne pendant une dizaine d'annees et requiert donc patience et determination.

15 De même, en amour, la réitération du désir sexuel entraîne en s'accomplissant d'infinies et délicieuses variations. 
matin. Dans ce cadre, un rythme peut aussi marquer le retour plaisant, et non accablant, du même, à l'instar du plaisir d'une habitude que l'on a soi-même instituée.

À égale distance d'une vie inerte, dont on souhaiterait s'abstraire, et d'une vie suractive sans aucun interstice libre, l'existence se déploie elle-même comme une alternance de mouvement et de repos. Bachelard montre en effet que, pour un être vivant, l'activité ne saurait être permanente, "lui succède toujours une période de nonfonctionnement, puisque l'énergie diminue dès qu'elle se dépense" (BACHELARD, 1993, p. 23). Même lorsqu'on a une impression de continuité, celle-ci est en réalité reconstruite, ou construite après coup: "l'expérience fine nous ramène au rythme des oui et des non, à la vie essayée, éphémère, refusée, reprise" (BACHELARD, 1993, p. 24).

En cela, l'existence suit la dynamique fondamentale du désir. Le désir lui-même et en lui-même est un rythme qui alterne entre mouvement et repos. Le désir, comme l'a bien montré Spinoza, est moins un manque qu'une puissance, un conatus ou puissance d'être, c'est-à-dire une puissance d'agir du corps et une puissance de penser de l'esprit. Quelle que soit son activité, l'homme est une unité biface corps/esprit: l'artisan ne laisse pas sa conscience au vestiaire et l'écrivain n'économise pas son énergie physique. Puissance qui porte l'existence, le désir est mouvement vers un objet, une visée, un but ; et repos lorsqu'il se réalise. Ce repos n'est pas morne atonie, mais le moment où s'éprouve une certaine satisfaction, une forme de réjouissance, une joie écrit Spinoza.

Les auteurs pessimistes exècrent le repos, symbole emblématique du temps où règne l'ennui, où se perçoivent le vide et la vanité de l'existence. "Comme le besoin pour le peuple, l'ennui est le tourment des classes supérieures. II a dans la vie sociale sa représentation le dimanche; et le besoin, les six jours de la semaine" (SCHOPENHAUER, 1984, p. 396). Cette idée résonne étonnamment avec le consumérisme actuel qui ne tolère aucune respiration dans le cycle hebdomadaire. Le droit à la paresse revendiqué par Lafargue s'est transformé en un droit à consommer nuit et jour tous les jours de la semaine et de l'année dans une injonction au shopping permanent ${ }^{16}$.

16 La césure hebdomadaire s'est émancipée du champ religieux et relève à présent du droit international, au moins dans son principe. Dans la Déclaration Universelle des Droits de l'homme, 
Pourtant, les désirs de chacun s'expriment aussi dans les jours de repos: faire une grasse-matinée ou une promenade, aller pique-niquer ou au cinéma, passer du temps en famille ou s'isoler, ne rien faire sans vouloir à tout prix anxieusement s'occuper. Le vivant de lui-même et pour lui-même désire du repos. Et, selon Russell, "le bon usage du loisir, il faut le reconnaître, est le produit de la civilisation et de l'éducation" (RUSSELL, 2002, p. 23).

Ce bon usage n'est pas accessible à tous, sinon en droit, du moins en fait. Mais dans un monde où personne ne serait contraint de travailler plus de quatre heures par jour, l'auteur envisage : "tous ceux qu'anime la curiosité scientifique pourront lui donner libre cours, et tous les peintres pourront peindre sans pour autant vivre dans la misère en dépit de leur talent. Les jeunes auteurs ne seront pas obligés de faire de la réclame en écrivant des livres alimentaires à sensation, en vue d'acquérir l'indépendance financière que nécessitent les œuvres monumentales qu'ils auront perdu le goût et la capacité de créer quand ils seront enfin libres de s'y consacrer" (RUSSELL, 2002, p. 36). Cette perspective, visionnaire dans les années 1930, est désormais économiquement réalisable, et urgemment si l'on souhaite résoudre sérieusement la question du chômage.

Russell, à rebours du classique jugement moralisateur condamnant l'oisiveté, met au centre de sa réflexion la qualité de l'existence : "le bonheur et la joie de vivre prendront la place de la fatigue nerveuse, de la lassitude et de la dyspepsie. II y aura assez de travail à accomplir pour rendre le loisir délicieux mais pas assez pour conduire à l'épuisement. Comme les gens ne seront pas trop fatigués dans leur temps libre, ils ne réclameront pas pour seuls amusements ceux qui sont passifs et insipides" (RUSSELL, 2002, p. 37). On le voit, l'enjeu autour de l'articulation du temps de travail et des loisirs n'est pas seulement social, économique et politique.

Une vie accordée au rythme de désirs essentiels est en effet riche et intense, le sujet y perçoit son pouvoir créateur, notamment des rythmes de son existence, dans une forme d'adéquation à soi. Il peut éprouver ce joyeux sentiment d'exister qu'une vie en bonne santé ne saurait à elle seule parvenir à susciter, un sentiment renforcé par l'engagement dans une activité créatrice.

l'article 24 édicte le droit au repos qui complète le droit au travail énoncé dans l'article 23 qui le précède. Cependant, le droit du travail s'acharne pour sa part à grignoter ce droit à ne pas travailler. 


\section{Les Rythmes De La CREATion}

Créer une œuvre, quelle qu'elle soit, artistique, philosophique, scientifique... instaure des rythmes particuliers dans l'existence. Certes, un créateur est monomaniaque, et il semblerait que la question des rythmes ne se pose même plus pour lui : il s'agit de créer, encore créer, toujours créer. Mais, porté par cet élan créateur, le sujet vit précisément aux rythmes de sa création car créer requiert du temps. La création se déploie sur une certaine durée qui, selon les domaines, varie par exemple de quelques heures pour une peinture à des années pour un livre. Elle se décompose en différents moments : celui de la formulation d'une nouvelle idée qui va constituer le moteur de la création ; celui, parfois long et laborieux, de la recherche d'éléments, de matériaux, de références, de connaissances pour nourrir ce projet ; celui du commencement effectif de la réalisation de cette œuvre et de sa délicate élaboration; et enfin celui de l'aboutissement de l'ensemble de ce processus par l'achèvement de l'œuvre. Des lenteurs et des moments de latence où rien ne semble pouvoir aboutir alternent avec des moments d'accélération et de fulgurance où tout semble s'agencer à merveille dans l'œuvre en cours.

Lorsqu'une œuvre est terminée, le ressort créateur marque quelque temps d'arrêt. Ce dernier peut être fort bref, le sujet passant d'une œuvre à une autre à une cadence soutenue. Ce temps du repos est toutefois plus important qu'il n'y paraît car il permet prise de distance, réflexion, questionnement, ressourcement qui sont tous nécessaires pour maintenir une certaine inventivité. Une suspension temporaire peut en effet s'avérer fertile. Parfois, une idée, une solution, une ouverture inattendue surgissent lors d'une marche ou d'une activité ménagère car la disponibilité d'esprit favorise cette émergence. Prenant régulièrement des années sabbatiques, le designer Stefan Sagmeister constate que de telles parenthèses sont très souvent suivies de périodes de profond renouvellement créatif ${ }^{17}$.

Une fois achevée, l'œuvre créée scelle la fin d'un dynamisme qui est celui-là même de l'acte qui l'a portée, elle existe objectivement et non plus à titre de projet, d'idée, d'intuition, de recherche en cours. "C'est dans la forme même du solide, de la cristallisation, de la permanence de l'existence, que l'esprit - devenu ainsi objet -

17On peut le constater au regard de la réalisation de l'identité visuelle de la Casa da Mùsica de Porto, par exemple. 
s'oppose au flux de la vie qui s'écoule, aux diverses tensions du psychisme subjectif" (SIMMEL, 1988, p. 177). Cette inscription dans une forme objective représente une transformation de ce qui est mouvant en une forme repérable et identifiable.

Ce rapport des œuvres au flux du vivant peut être envisagé comme une fixation problématique. "La vie frémissante se développant à l'infini, créatrice, dans quelque sens que ce soit, voit se dresser en face d'elle sa propre production, ferme, avec l'inquiétant effet de retour de fixer cette vivacité, de la figer ; on dirait souvent que la mobilité féconde de l'âme meurt par sa propre production" (SIMMEL, 1988, p. 184). L'œuvre représente sous un certain aspect la négation du vivant, mais cette négation est l'expression d'un véritable renversement car, si on y regarde de plus près, les supposés défauts de la fixation en une œuvre se retournent en qualités : face à un flux, la délimitation ; face au changement, le stable et le durable ; face à une certaine dispersion, la cohésion et la cohérence que porte une œuvre avec elle.

La vie toujours mouvante, aussi rythmée soit-elle, peine à accéder à de telles qualités alors que l'œuvre, quelle qu'elle soit, permet de coaguler le mouvement, sa réalisation est ainsi un événement qui rythme objectivement le temps subjectif, ce qui n'est pas rien. L'œuvre, qui vivra ensuite sa vie, permet au sujet de s'appréhender et d'exister en tant que créateur. Comme l'écrit Robert Misrahi, "certes, il existe une dialectique malheureuse de l'auteur et de l'œuvre, par laquelle celle-ci échappe à celuila par son caractère même de détermination et d'objectivité. Mais cette dialectique ne saurait nous faire oublier le moment inverse, lequel consiste, pour l'œuvre, à donner une réalité à l'auteur" (MISRAHI, 1969, p. 215). C'est un fait, l'auteur gagne d'exister comme origine et source d'une œuvre qui existe face à lui, sa vie prend corps et se structure à partir d'elle.

La création instaure un dynamisme qui ne s'arrête pas à lui-même, elle ouvre sur un avenir qui sera lui-même créateur ; cette fécondité dépend de l'investissement du sujet qui doit sans cesse motiver la poursuite d'une activité qui repose in fine sur son seul désir d'être et de demeurer créateur.

L'attitude créatrice peut en effet s'user, décliner, perdre de son dynamisme, se bloquer. Gorz y voit la marque du vieillissement : "C'est au commencement seulement (d'une vie, d'une entreprise, d'un couple...) que les fins déterminent les moyens à inventer, que le projet façonne le monde à l'image d'un but qui est absence. À mesure que vous avancez, les moyens forgés perpétuent dans l'inertie de leur matière la 
finalité première (et souvent déjà morte) de vos actes passés" (GORZ, 2004, p. 404). Cette inertie pousse un homme à devenir une pâle imitation de lui-même.

II est vrai que tout commencement insuffle un nouveau rythme à l'existence. Mais il n'y a aucune raison de penser que cette possibilité concerne la seule jeunesse car on peut être jeune et apathique alors qu'on peut, a contrario, passer sa vie à entreprendre jusqu'à un âge avancé, avec la même verve, de nouveaux projets, tout en se fondant sur des projets bien étayés menés à leur terme. II est juste néanmoins, comme le remarque Bachelard, qu'à un moment donné, l'esprit conservatif ou académique peut dominer. "C'est verser dans un vain optimisme que de penser que savoir sert automatiquement à savoir, que la culture devient d'autant plus facile qu'elle est plus étendue, que l'intelligence enfin, sanctionnée par des succès précoces, par de simples concours universitaires, se capitalise comme une richesse matérielle" (BACHELARD, 1986, p. 15). Il y a un moment où l'esprit préfère ce qui le conforte à ce qui pourrait le bousculer - un temps "où il aime mieux les réponses que les questions" (BACHELARD, 1986, p. 15). II se contente alors de ressasser ses acquis car demeurer créatif réclame un effort constant.

Le renouvellement n'est pas pour autant le signe d'une insatisfaction permanente et de l'échec du désir à atteindre ses buts. On peut y voir plus simplement un dynamisme sans cesse orienté vers l'avenir, non parce que l'insatisfaction croît à mesure que des œuvres sont créées, mais parce que les œuvres novatrices suscitent une continuation de l'acte créateur sous de nouvelles modalités. La relance permanente du désir de créer exprime la vitalité d'un désir renaissant qui, en atteignant des buts, souhaite pourtant en poursuivre et en atteindre de nouveaux. Nul cercle qui ramènerait toujours au même point, à l'image d'un serpent qui se mord la queue, mais un mouvement en spirale où ce qui est à faire se nourrit de ce qui a été fait.

L'existence est rythmée par ces créations successives auxquelles il faut ajuster la vie quotidienne, matérielle, familiale, sociale. L'écrivain Arnauld Lebrusq résout ainsi la difficile équation entre travail et création : on voit, comme il le dit, trop de gens malheureux à vouloir "s'épanouir" au travail alors que le travail devrait parfois être pris comme un simple moyen d'assurer sa subsistance. Une fois cette question réglée, on peut s'adonner avec enthousiasme à la création.

Une vie où la création a une place centrale comporte des temps forts: une série de concerts, une exposition, une publication, une découverte, un cycle de conférences. 
Ces temps sont forts, tant du point de vue affectif, par ce qui s'y joue, que du point de vue du sens de ce qui est vécu. Concentrant une extrême créativité, ces moments permettent de se sentir intensément vivant.

Le temps de la création est lui-même vécu d'une façon particulière: "c'est un temps qui oublie le temps, pendant lequel le temps ne (se) compte plus. II advient ou survient quand une activité apporte une plénitude. [...] Cette activité s'accorde à ellemême et au monde. Elle a quelques traits d'une autocréation et d'un don plutôt que d'une obligation ou d'une imposition venue du dehors" (LEFEBVRE et REGULIER, 1985, p. 194).

La création institue un jeu nouveau dans l'existence par lequel le sujet peut accéder à une forme de stabilité existentielle. En effet, les questions qui taraudent un créateur concernent surtout les problèmes posés par des projets particuliers, les difficultés de leur réalisation, alors que sur le fond se fortifie son désir de poursuivre son activité créatrice. Chaque création poursuit des intérêts particuliers, mais ces actes apparaissent aussi comme une multiplicité de voies grâce auxquelles un sujet s'accomplit. Les événements exceptionnels, quant à eux, s'intègrent plus aisément dans l'existence, qu'ils soient heureux, comme une belle rencontre, une nouvelle amitié, une proposition renforçant des engagements ou malheureux, comme une rupture amoureuse, la mort d'un proche, l'éloignement d'un ami. Ces derniers, lorsqu'ils s'inscrivent dans une existence solidement arrimée, sont vécus pour ce qu'ils sont, des moments douloureux.

Vivre au rythme de ses créations, c'est déployer son existence sous la forme d'une synthèse paradoxale, celle du mouvement et du repos. L'existence, délestée de l'agitation et d'une certaine impatience, s'éprouve à la fois comme un dynamisme qui est celui-là même du désir, et comme un repos qui est celui d'un désir atteignant des buts essentiels. L'existence est alors vécue, pour reprendre la belle formule de Bachelard, comme une vibration heureuse.

\section{CONCLUSION}

J'ai volontairement laissé de côté les rythmes se situant au sein d'une création donnée. II y a là de vastes champs de réflexion nécessitant d'être étudiés pour euxmêmes, en particulier dans le domaine artistique : musique, littérature, poésie, cinéma,... Se rendre sensible à l'esthétique des rythmes, c'est se rendre sensible à la dynamique du désir qui sous-tend toute activité humaine. L'essentiel était ici de 
montrer que s'engager dans la reconquête de ses propres rythmes permet de se construire soi-même. II serait temps d'en prendre conscience car, ultra-connecté de manière compulsive à des tablettes, des ordinateurs et des ordiphones de tous types, réagissant au rythme de leurs fréquentes alertes, mais déconnecté de lui-même, de l'attention aux autres et de sa présence au monde, l'homme contemporain sombre doucement, sans y prendre garde, dans un état d'extrême servitude volontaire. 


\section{RÉFÉRENCES}

BACHELARD, Gaston. La Formation de l'esprit scientifique. Paris : Vrin, 1986. . La Dialectique de la durée. Paris : Quadrige/PUF, 1993.

BÉLOT Laure, "La dictature de l'impatience". In : Le Monde, Science \& médecine, Mercredi 26 juin 2019.

BERGSON, Henri. L'Évolution créatrice. Paris : Quadrige/PUF, 1981.

CAMUS, Albert. Le Premier homme, Paris : Gallimard, 1994.

CANGUILHEM, Georges. Le Normal et le pathologique. Paris : Quadrige/PUF, 1998.

GEOFFROY, Emmanuel. L'imam gay, Reportage de Llana Navaro. Documentaire radiophonique, Les Pieds sur terre, Sonia Kronlund, France Culture, 19. 11. 2013 (28 min).

GORZ, André. Le Traître suivi de Le vieillissement. Paris : Folio/essais, Gallimard, 2004.

LEFEBVRE, Henri. Éléments de rythmanalyse, Introduction à la connaissance des rythmes. Paris : Syllepse, 1992.

LEFEBVRE Henri et RÉGULIER Catherine. "Le projet rythmanalytique". In : Communications, $\mathrm{N}^{\circ} 41$, p. 191-199, 1985.

MISRAHI, Robert. Lumière, commencement, liberté. Paris : Plon, 1969.

La Jouissance d'être. Le sujet et son désir, essai d'anthropologie

philosophique. La Versanne : Encre Marine, 1996.

RUSSELL, Bertrand. Éloge de l'oisiveté. Paris : Allia, 2002.

SAGMEISTER, Stefan. Le pouvoir du temps libre. Conférence filmée, TEDGlobal 2009, (17 $\min 40)$.

SCHOPENHAUER, Arthur. Le Monde comme volonté et comme représentation. Paris : PUF, 1984.

SICARD Didier. "La médecine sans le corps : quelques notes sur la relégation du corps". In : Les Cahiers du Centre Georges Canguilhem, 2007/1 (Nº1), PUF, p. 113-137.

SIMMEL, Georg. La Tragédie de la culture et autres essais. Paris : Rivages, 1988.

SPINOZA. Éthique. Paris : Le Livre de Poche, 2005. 
THOMAS, Jean-Paul. La Médecine, nouvelle religion. Paris : François Bourin Éditeur, 2013.

VELUT, Stéphane. L'Hôpital, une nouvelle industrie. Le langage comme symptôme, Paris : Tracts/Gallimard, 2020.

VERDIER, Véronique. Existence et création, Paris : L'harmattan, 2016. 


\section{Os RITMOS DA EXISTÊNCIA}

\section{RESUMO}

Este artigo esboça o itinerário de reconquista dos ritmos da existência. Partindo de uma constatação, a existência é, de fato, ritmada, mas esses ritmos são geralmente experenciados passivamente e não escolhidos pelo sujeito, nós nos perguntamos como reencontrar uma mestria desses ritmos posto que esta mestria é uma condição para viver melhor, ou mesmo para bem viver. Da escuta do corpo, posto em perigo pela ruptura de uma doença, passando pela imposição de regras rígidas, veremos que a saída se situa na criação de ritmos próprios.

PalaVRAS-ChaVe: Existência; Ritmos; Criação; Desejo; Spinoza; Bachelard; Canguilhem; Lefebvre. 


\title{
The RHYTHMS Of EXISTENCE
}

\begin{abstract}
This article sketches the itinerary of regaining the rhythms of the existence. Starting from an observation, the existence is in fact rhythmic but these rhythms are most often undergone and not chosen by the subject, we wonder how to find again a mastery of these rhythms, since this mastery is one condition to live better, maybe even to live well. From the listening of the body, endangered by the rupture of an illness, passing by the constraint of rigid rules, we will see that the exit lies in the creation of one's own rhythms.
\end{abstract}

KeYWORDS: Existence; Rhythm; Creation; Desire; Spinoza; Bachelard; Canguilhem; Lefebvre. 
RECEBIDO EM 10/12/2019

APROVADO EM 14/04/2020

(C) 2020 Psicanálise \& Barroco em revista

http://www.seer.unirio.br/index.php/psicanalise-barroco/index revista@psicanaliseebarroco.pro.br

Programa de Pós-Graduação em Memória Social — UNIRIO

Memória, Subjetividade e Criação

www.memoriasocial.pro.br/proposta-area.php 